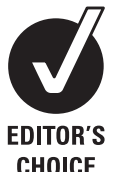

CHOICE

ORIGINAL ARTICLE

\title{
Prior antiplatelet or anticoagulant therapy and mortality in stroke
}

\author{
Chun Shing Kwok, ${ }^{1}$ Jane Skinner, ${ }^{2}$ Anthony K Metcalf, ${ }^{1}$ John F Potter, ${ }^{1,2}$ \\ Phyo Kyaw Myint ${ }^{1,2,3}$
}

See Editorial, p 677

- Additional materials are published online only. To view these files please visit the journal online (http://heart.bmi. com/content/98/9.toc).

${ }^{1}$ Stroke Research Group, Norfolk and Norwich University Hospital, Norwich, UK

${ }^{2}$ Norwich Medical School, Faculty of Medicine and Health Sciences, University of East Anglia, Norwich, UK

${ }^{3}$ Clinical Gerontology Unit, University of Cambridge, Cambridge, UK

\section{Correspondence to} Dr Phyo Kyaw Myint, Norwich Medical School, Chancellors Drive, University of East Anglia, Norwich, Norfolk NR4 7TJ, UK; phyo.k.myint@uea.ac.uk

Accepted 18 January 2012 Published Online First 10 February 2012

\author{
ABSTRACT \\ Objective To examine the influence of previous \\ antiplatelet or anticoagulant therapy on subsequent \\ stroke mortality at different time points up to 1 year post \\ stroke.
}

Design Data were examined from a hospital register collected over 5 years (2004-2008).

Setting A single large university hospital.

Participants Every adult (18+ years) admitted with an acute stroke.

Main outcome measures Mortality risks at 7, 30, 60, 90 and 365 days were assessed using logistic regression following ischaemic and haemorrhagic stroke, adjusting for age, gender, premorbid Rankin and stroke type.

Results 3308 patients with first or recurrent stroke were included ( $53 \%$ women, mean age $77.7 \pm 11.5$ years, $86 \%$ ischaemic stroke). One-year mortality was $35.2 \%$ (999) for ischaemic stroke and $48.3 \%$ (227) for haemorrhagic stroke. Compared with no previous therapy, the mortality following ischaemic stroke for those already receiving antiplatelets or anticoagulants was not associated with increased mortality at any time points up to 1 year after presentation in the fully adjusted model. However, patients with haemorrhagic stroke had a worse prognosis at all time points after standard risk factor adjustment. For patients who used aspirin or warfarin prior to haemorrhagic stroke compared with no use, ORs (95\% Cls) were 1.31 (0.64 to 2.68) and 2.91 (1.23 to 6.89) for 7 days, 2.36 (1.18 to 4.71$)$ and 2.37 (1.00 to 5.61) for 30 days, 2.18 (1.10 to 4.29) and 2.86 (1.20 to 6.84$)$ for 60 days, 2.56 (1.27 to 5.13) and 2.82 (1.16 to 6.86$)$ for 90 days and 1.67 (0.89 to 3.12$)$ and 2.44 (1.06 to 5.62) for 365 days.

Conclusions Prior antiplatelet or anticoagulant use was associated with increased mortality following HS but not IS after adjustment for common factors associated with a poor prognosis. The reasons for this poor prognosis and potential therapeutic options need exploring in future studies.

\section{INTRODUCTION}

Antiplatelet/anticoagulant (AP/AC) agents are frequently prescribed for people at risk of stroke and other cardiovascular diseases in primary and secondary prevention. Good evidence exists for the use of these agents in secondary prevention following ischaemic stroke $e^{1}$ but their use in primary prevention especially for AP agents remains controversial. ${ }^{3} 4$

Apart from warfarin use in atrial fibrillation, the effect of these agents prior to stroke on subsequent survival is not well known. To date, conflicting evidence exists regarding the influence of premorbid medications on outcomes in patients with ischaemic stroke. ${ }^{5}{ }^{6}$ Similarly there is mixed evidence regarding the impact of prior AP agents in patients with haemorrhagic stroke. Foerch et al, using data from the German Stroke Register, showed that pretreatment with AP agents was not independently associated with unfavourable outcomes, ${ }^{7}$ although a recent meta-analysis suggested it was associated with an increase in mortality but not poor functional outcomes. ${ }^{8}$

Whether AP/AC use prior to stroke is associated with an increased or a decreased mortality risk and whether this is influenced by the time from stroke onset has not been previously examined. In this study, we investigate the effects on mortality at 7 , $30,60,90$ and 365 days post stroke in relation to previous $\mathrm{AP} / \mathrm{AC}$ therapy for ischaemic and haemorrhagic stroke.

\section{METHODS}

The study sample consists of consecutive stroke patients admitted to a single large university hospital with a catchment population of approximately 750000 in Central Norfolk, UK between January 2004 and November 2008. The study participants were drawn from the comprehensive Norfolk and Norwich University Hospital Stroke Register, which has been previously described. ${ }^{9}$ Briefly, the Norfolk and Norwich Hospital Stroke Register was established in late 1996 based on the European Basic Stroke Register and collects information on every adult (18+ years) admitted with an acute stroke. Institutional approval was obtained from the Norfolk and Norwich Hospital Trust Research and Development Committee.

\section{Data collection}

Data were collected prospectively by specialist stroke nurses or doctors to ascertain variables such as age, gender, type of stroke based on CT (ischaemic or haemorrhagic), premorbid Rankin score (0-5), Oxfordshire Community Stroke Project (OCSP) Classification and mortality. Haemorrhagic transformation of ischaemic stroke was classified as ischaemic stroke and subarachnoid haemorrhages were excluded. All stroke cases were verified by experienced stroke physicians. Information on prior AP/AC use was obtained from the medication list of the patient on admission. For each patient, when admitted the premorbid (prestroke) Rankin score (see footnote to table 1), as modified by the UK transient ischaemic attack 
Table 1 Sample characteristics by type of prior antiplatelet and anticoagulant use

\begin{tabular}{|c|c|c|c|c|c|c|}
\hline & Total & None & Aspirin & Aspirin plus dipyridamole & Clopidogrel & Warfarin \\
\hline Patients & 3308 & $1763(53)$ & $1140(34)$ & $94(3)$ & $74(2)$ & $237(7)$ \\
\hline \multicolumn{7}{|l|}{ Age group } \\
\hline$<65$ & 447 (14) & $317(18)$ & $98(9)$ & $6(6)$ & 10 (14) & $16(7)$ \\
\hline $75-84$ & $1300(39)$ & $641(36)$ & $455(40)$ & $53(56)$ & $29(39)$ & $134(51)$ \\
\hline$>85$ & $908(27)$ & $444(27)$ & $372(33)$ & $22(23)$ & $17(23)$ & $53(22)$ \\
\hline \multicolumn{7}{|l|}{ Gender } \\
\hline \multicolumn{7}{|l|}{ Premorbid Rankin* } \\
\hline 0 & $2161(70)$ & $1206(73)$ & $706(66)$ & $49(57)$ & $48(70)$ & $152(70)$ \\
\hline 1 & $236(8)$ & $110(7)$ & $81(8)$ & $16(19)$ & $5(7)$ & $24(11)$ \\
\hline 2 & $216(7)$ & $107(7)$ & $84(8)$ & $5(6)$ & $6(9)$ & $14(6)$ \\
\hline 3 & $298(10)$ & $139(8)$ & 127 (12) & 10 (12) & $7(10)$ & $15(7)$ \\
\hline \multicolumn{7}{|l|}{ OCSP classification } \\
\hline TACS & $809(26)$ & $366(22)$ & $259(24)$ & $28(32)$ & $17(24)$ & $58(27)$ \\
\hline PACS & $1053(34)$ & $577(35)$ & $359(33)$ & $26(30)$ & $28(39)$ & $63(29)$ \\
\hline POCS & $489(16)$ & $430(26)$ & $165(15)$ & $23(26)$ & $12(17)$ & $34(16)$ \\
\hline LACS & $721(23)$ & $267(16)$ & $366(22)$ & $11(13)$ & $14(20)$ & $54(25)$ \\
\hline Unspecified & $49(2)$ & $27(2)$ & 27 (1) & $0(0)$ & $0(0)$ & $6(3)$ \\
\hline
\end{tabular}

Data presented are number (\%).

*Premorbid Rankin: 0: no symptoms at all; 1: no significant disability despite symptoms, able to carry out all usual duties and activities; 2: slight disability; unable to carry out all previous activities but able to look after own affairs without assistance; 3 : moderate disability, requiring some help, but able to walk without assistance; 4 : moderately severe disability, unable to walk without assistance and unable to attend to own bodily needs without assistance; 5 : severe disability, bedridden, incontinent and requiring constant nursing care and attention.

LACS, lacunar stroke; OCSP, Oxfordshire Community Stroke Project; PACS, partial anterior circulation stroke; POCS, posterior circulation stroke; TACS, total anterior circulation stroke.

investigators, ${ }^{10}$ was ascertained from nursing and medical records. For this study, data were included up to November 2008 with 1-year follow-up data ascertained in November 2009. Prior AP/AC use was recorded since January 2004 and any stroke patients admitted up to that time point were excluded.

\section{Outcome}

The outcome of interest was mortality in the short (7 and 30 days), medium (60 and 90 days) and long term (365 days) post admission for the index stroke admission. Whether patients were dead or alive at these time points was ascertained using the hospital Patient Administrative System (PAS), which has been previously described. ${ }^{11}$ Because nearly $100 \%$ of the population in the UK is registered with the National Health Service (NHS), the PAS which records the healthcare episodes is a valid and reliable way of determining mortality. Briefly, PAS records the movement of patients and whether a patient is alive or dead at any given time by having a system that enables local general practitioners to report deaths that occur in the community.

\section{Statistical analysis}

Data were analysed using STATA V.10.0 (2009, StataCorp). Kaplan-Meier (KM) curves (unadjusted) were drawn for mortality based on different prior $\mathrm{AP} / \mathrm{AC}$ medication use. Logistic regression was used rather than survival analysis methods because there were no competing risks as we analysed all-cause mortality and had a follow-up rate of $100 \%$ for at least a year, so there was no censoring.

Risk estimates (ORs) were determined using four different models: first unadjusted, second adjusting for age, the square of age and gender, third additionally adjusting for premorbid Rankin score, and finally also adjusting for Oxfordshire
Community Stroke Project (OSCP) classification. Age was modelled as a quadratic term because the age-mortality relationship was nonlinear. Because discharge AP/AC medication depends on the admission drug therapy and patients' condition (eg, newly diagnosed atrial fibrillation), we repeated the analyses by additionally adjusting for discharge $\mathrm{AP} / \mathrm{AC}$ use for those who were discharged alive after ischaemic stroke.

\section{RESULTS}

A total of 3308 patients were included in this study. Table 1 shows the characteristics of these patients by different prestroke medication. The majority (51\%) of patients were not on any $\mathrm{AP} / \mathrm{AC}$ treatment prior to stroke. Among those who were on $\mathrm{AP} / \mathrm{AC}$ medication, aspirin was the most commonly used therapy (34\%). Of the study population, $53 \%$ were women, $86 \%$ presented with ischaemic stroke and $14 \%$ presented with haemorrhagic stroke.

Table 2 shows patient mortality for the various follow-up time points. The highest mortality following ischaemic stroke was observed between 90 and 365 days post ictus. In contrast, for haemorrhagic stroke the highest mortality occurred in the first 7 days following the incident stroke. The cumulative mortality was significantly lower for patients with ischaemic stroke compared with haemorrhagic stroke at 1 year. A log-rank test for equality of the survivor functions showed a highly significant difference between the groups over 365 days of follow-up.

The $\mathrm{K}-\mathrm{M}$ survival curves for the patients who were receiving different $\mathrm{AP} / \mathrm{AC}$ regimes (warfarin in $7 \%$ of cases) are shown in figures 1 and 2 for haemorrhagic and ischaemic stroke, respectively. For haemorrhagic stroke, prior aspirin or warfarin use was associated with reduced survival compared with no prior aspirin 
Table 2 Mortality of patients between different time points

\begin{tabular}{|c|c|c|c|c|c|c|c|c|c|}
\hline \multirow[b]{2}{*}{ Time points } & \multicolumn{4}{|c|}{ Ischaemic stroke } & \multicolumn{4}{|c|}{ Haemorrhagic stroke } & \multirow[b]{2}{*}{$\begin{array}{l}\text { p Value } \\
\text { (Ischaemic vs } \\
\text { haemorrhagic) }\end{array}$} \\
\hline & $\begin{array}{l}\text { No. } \\
\text { at risk }\end{array}$ & $\begin{array}{l}\text { No. } \\
\text { deaths (\%) }\end{array}$ & $\begin{array}{l}\text { Risk of } \\
\text { mortality (chance } \\
\text { in 1000)/day }\end{array}$ & $\begin{array}{l}\text { Cumulative mortality } \\
\text { function }(\%)(95 \% \mathrm{Cl})\end{array}$ & $\begin{array}{l}\text { No. } \\
\text { at risk }\end{array}$ & $\begin{array}{l}\text { No. } \\
\text { deaths (\%) }\end{array}$ & $\begin{array}{l}\text { Risk of } \\
\text { mortality (chance } \\
\text { in 1000)/day }\end{array}$ & $\begin{array}{l}\text { Cumulative } \\
\text { mortality function } \\
(\%)(95 \% \text { CI) }\end{array}$ & \\
\hline $8-30$ days & 2637 & $285(11)$ & 15.4 & 17.1 (15.8 to 18.6$)$ & 348 & $55(16)$ & 22.6 & 37.7 (33.5 to 42.2 ) & 0.0040 \\
\hline $31-60$ days & 2352 & $160(7)$ & 9.7 & 22.8 (21.3 to 24.4$)$ & 293 & $22(8)$ & 10.7 & 42.3 (38.0 to 46.9$)$ & 0.7812 \\
\hline $61-90$ days & 2192 & $71(3)$ & 4.6 & 25.3 (23.7 to 26.9$)$ & 271 & $6(2)$ & 3.2 & 43.6 (39.3 to 48.2 ) & 0.3182 \\
\hline
\end{tabular}

or warfarin at all follow-up time points. For ischaemic stroke, at no time point was there a statistically significant difference between any drug type compared with no prior treatment.

For ischaemic stroke, the risk of mortality by prior use of AP/ $\mathrm{AC}$ is shown in table 3 at various time intervals $(0-7$ days, 0-30 days, $0-60$ days, $0-90$ days and $0-365$ days). At no time point was there a statistically significant difference between each type of drug compared with no prior treatment. The risk of mortality by prior use of aspirin and warfarin in haemorrhagic stroke is shown in table 4. At all time points evaluated, the use of warfarin was associated with a statistically significant risk of mortality compared with no therapy. The risk was highest in the immediate post-stroke period up to 60 days, although similar ORs were observed at all time points, with all point estimates showing a greater than 2.3-fold increase in the odds of dying for prior warfarin use compared with no therapy using the fully adjusted model (model C). A similar pattern was observed for aspirin between 1 and 3 months post stroke (30, 60 and 90 days).

We present the discharge AP/AC medications based on prior use of these medication for ischaemic and haemorrhagic stroke in online supplementary tables 1 and 2, respectively. Repeating the analyses adjusting for discharge AP/AC medication for those who were discharged alive did not alter the results for those with ischaemic stroke (online supplementary table 3).

We excluded 323 patients who did not have radiological confirmation of stroke diagnosis because these patients were extremely unwell and the clinicians chose palliation (supportive care only) without further radiological investigation. There was a $98 \%$ mortality rate among these patients. Repeating the analyses after including them based on clinical diagnosis did not alter the results (data not shown).

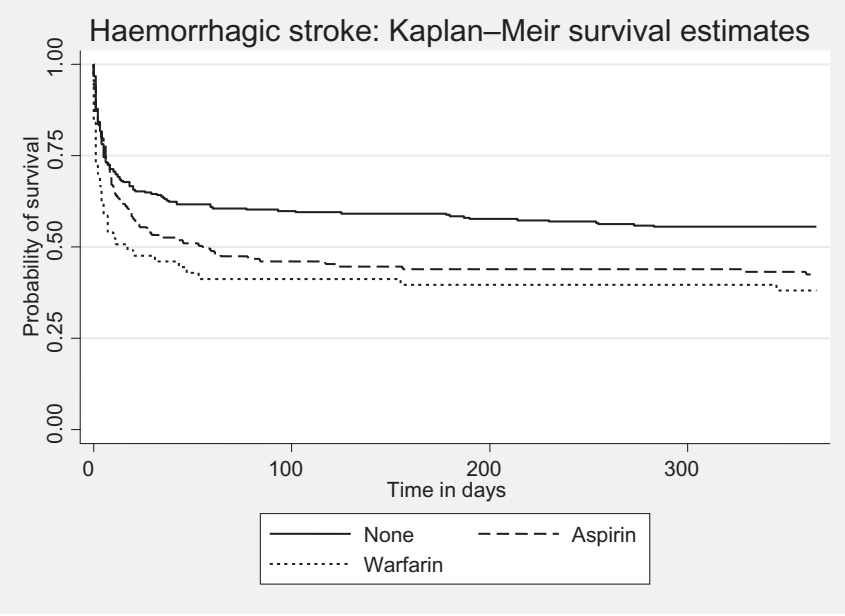

Figure 1 Kaplan-Meier survival estimates for patients with haemorrhagic stroke by prior antiplatelet or anticoagulant use.

\section{DISCUSSION}

This study showed no evidence that short, medium or longer term mortality (up to 1 year) post stroke was influenced by prior $\mathrm{AP} / \mathrm{AC}$ therapy in patients who had ischaemic stroke. However, in patients with haemorrhagic stroke, prior use of warfarin poses a significantly increased risk of mortality at all time points examined. Prior use of aspirin therapy also appears to carry a higher risk compared with no therapy at 30, 60 and 90 days in this group.

Our results suggest that there is an increase in early mortality (within 7 and 30 days) for haemorrhagic stroke associated with warfarin therapy. Previous studies of patients on aspirin therapy prior to intracranial haemorrhage found similar poor short-term outcomes and increased mortality, and suggested that it may be related to haematoma growth in patients on AP therapy. ${ }^{12} 13$ However there are other studies suggesting that AP medication at the onset of intracerebral haemorrhage is not associated with an increase in haemorrhage volume, haemorrhage expansion and clinical outcome at 90 days. $^{14}$ An ongoing clinical trial is exploring the potential benefit of platelet transfusion in patients with prior AP therapy and intracerebral haemorrhage. ${ }^{15}$

To our knowledge, this is the first study to report the effects of prior AP/AC treatment on mortality at different time points for both short ( 7 and 30 days), medium (60 and 90 days) and longer-term follow-up (365 days) after hospital admission with acute ischaemic or haemorrhagic stroke. While Thompson and colleagues previously reported that AP use was associated with an increased risk of mortality in haemorrhagic stroke in their meta-analysis up to 90 days post stroke, ${ }^{8}$ we have furthered their observations in that prior warfarin use also increases the risk of mortality post haemorrhagic stroke up to 1-year

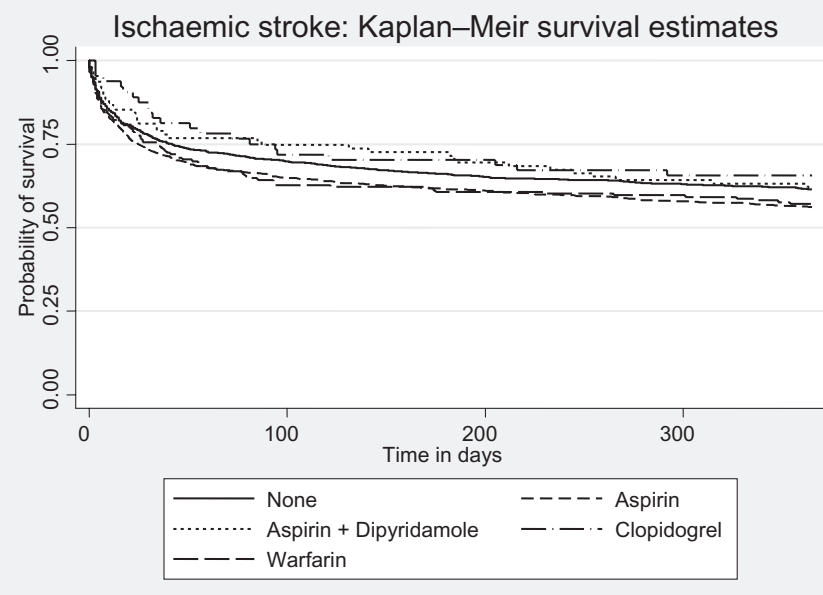

Figure 2 Kaplan-Meier survival estimates for patients with ischaemic stroke by prior antiplatelet or anticoagulant use. 
Table 3 ORs ( $95 \%$ Cls) of mortality post ischaemic stroke based on prior therapy

\begin{tabular}{|c|c|c|c|c|}
\hline Mortality & Unadjusted & Model A & Model B & Model C \\
\hline $0-7$ days, $\mathrm{N}$ & 2838 & 2838 & 2654 & 2530 \\
\hline No therapy* & 1.00 & 1.00 & 1.00 & 1.00 \\
\hline Aspirin & $1.07(0.78$ to 1.46$)$ & $0.99(0.73$ to 1.36$)$ & $1.05(0.74$ to 1.48$)$ & $0.92(0.64$ to 1.34$)$ \\
\hline Aspirin plus dipyridamole & $1.03(0.44$ to 2.41$)$ & 0.95 (0.40 to 2.23$)$ & $1.18(0.50$ to 2.83$)$ & $0.94(0.38$ to 2.37$)$ \\
\hline Clopidogrel & $0.93(0.33$ to 2.61$)$ & $0.96(0.34$ to 2.71$)$ & $1.26(0.44$ to 3.59$)$ & $1.79(0.59$ to 5.45$)$ \\
\hline Warfarin & $1.32(0.77$ to 2.30$)$ & $1.29(0.74$ to 2.25$)$ & 1.28 (0.69 to 2.38$)$ & $1.40(0.72$ to 2.72$)$ \\
\hline $0-30$ days, $\mathrm{N}$ & 2838 & 2838 & 2654 & 2530 \\
\hline No therapy* & 1.00 & 1.00 & 1.00 & 1.00 \\
\hline Aspirin & $1.18(0.96$ to 1.46$)$ & $1.05(0.85$ to 1.30$)$ & $1.01(0.80$ to 1.28$)$ & $0.90(0.70$ to 1.17$)$ \\
\hline Aspirin plus dipyridamole & $0.84(0.45$ to 1.57$)$ & $0.73(0.39$ to 1.38$)$ & $0.75(0.38$ to 1.47$)$ & $0.60(0.29$ to 1.26$)$ \\
\hline Clopidogrel & $0.98(0.49$ to 1.94$)$ & 1.05 (0.52 to 2.13$)$ & 1.17 (0.56 to 2.48$)$ & $1.67(0.75$ to 3.71$)$ \\
\hline Warfarin & $1.15(0.77$ to 1.72$)$ & $1.09(0.73$ to 1.65$)$ & $1.02(0.65$ to 1.60$)$ & $0.88(0.52$ to 1.49$)$ \\
\hline $0-60$ days, $\mathrm{N}$ & 2838 & 2838 & 2654 & 2525 \\
\hline No therapy* & 1.00 & 1.00 & 1.00 & 1.00 \\
\hline Aspirin & $1.18(0.98$ to 1.43$)$ & $1.03(0.85$ to 1.26$)$ & 1.00 (0.81 to 1.23$)$ & $0.90(0.71$ to 1.14$)$ \\
\hline Aspirin plus dipyridamole & 0.77 (0.44 to 1.37$)$ & $0.66(0.37$ to 1.18$)$ & $0.69(0.38$ to 1.28$)$ & $0.55(0.28$ to 1.09$)$ \\
\hline Clopidogrel & $1.04(0.57$ to 1.92$)$ & $1.15(0.61$ to 2.16$)$ & $1.07(0.53$ to 2.15$)$ & $1.49(0.70$ to 3.16$)$ \\
\hline Warfarin & $1.32(0.93$ to 1.88$)$ & 1.25 (0.87 to 1.79$)$ & $1.23(0.83$ to 1.82$)$ & $1.20(0.76$ to 1.89$)$ \\
\hline $0-90$ days, $\mathrm{N}$ & 2838 & 2838 & 2654 & 2525 \\
\hline No therapy* & 1.00 & 1.00 & 1.00 & 1.00 \\
\hline Aspirin & $1.18(0.98$ to 1.42$)$ & $1.02(0.85$ to 1.24$)$ & $0.99(0.80$ to 1.21$)$ & $0.90(0.72$ to 1.14$)$ \\
\hline Aspirin plus dipyridamole & $0.79(0.46$ to 1.36$)$ & $0.66(0.38$ to 1.16$)$ & $0.71(0.40$ to 1.28$)$ & $0.58(0.30$ to 1.12$)$ \\
\hline Clopidogrel & $1.09(0.61$ to 1.94$)$ & 1.21 (0.66 to 2.21$)$ & $1.17(0.60$ to 2.26$)$ & $1.64(0.80$ to 3.34$)$ \\
\hline Warfarin & $1.40(1.00$ to 1.96$)$ & $1.31(0.93$ to 1.86$)$ & $1.26(0.86$ to 1.85$)$ & $1.28(0.82$ to 1.98$)$ \\
\hline $0-365$ days, $\mathrm{N}$ & 2838 & 2838 & 2654 & 2525 \\
\hline No therapy* & 1.00 & 1.00 & 1.00 & 1.00 \\
\hline Aspirin & $1.18(1.00$ to 1.40$)$ & $1.00(0.84$ to 1.19$)$ & $0.96(0.80$ to 1.16$)$ & $0.90(0.73$ to 1.10$)$ \\
\hline Aspirin plus dipyridamole & $0.96(0.60$ to 1.52$)$ & 0.78 (0.48 to 1.27$)$ & $0.81(0.49$ to 1.35$)$ & $0.66(0.37$ to 1.17$)$ \\
\hline Clopidogrel & $1.06(0.63$ to 1.80$)$ & $1.20(0.68$ to 2.10$)$ & $1.10(0.60$ to 2.02$)$ & $1.45(0.75$ to 2.78$)$ \\
\hline Warfarin & $1.22(0.89$ to 1.68$)$ & $1.10(0.79$ to 1.54$)$ & $1.10(0.77$ to 1.58$)$ & $1.06(0.71$ to 1.59$)$ \\
\hline \multicolumn{5}{|c|}{$\begin{array}{l}\text { Model A: adjusted for age, age squared, and gender. } \\
\text { Model B: adjusted for age, age squared, gender and premorbid Rankin score. } \\
\text { Model C: adjusted for age, age squared, gender, premorbid Rankin score and Oxfordshire Community Stroke Project (OCSP) stroke } \\
\text { type. }\end{array}$} \\
\hline
\end{tabular}

follow-up. We also found that the increased mortality risk in haemorrhagic stroke with the prior use of aspirin occurred at 30day and 90-day follow-up.

As a hospital-register-based study, we are not able to assess the benefit of use of warfarin to prevent ischaemic stroke at the population level. It is well known that warfarin use has an overall beneficial effect in preventing ischaemic stroke in those with atrial fibrillation at the expense of higher risk of major bleeding, including haemorrhagic stroke. ${ }^{16} 17$ Understanding the likelihood of mortality at different time points based on different stroke types and prior use of $\mathrm{AP} / \mathrm{AC}$ has clinical prognostic value. This information can be considered in conjunction with other risk factors for mortality to help patients, families and clinicians to decide on treatment options and appropriate care. ${ }^{18}$ One recent study evaluated stroke incidence and death rates using the Ludwigshafen Stroke Register. ${ }^{19}$ This study reported first-ever stroke death rates of $13.6 \%, 16.4 \%$ and $23.3 \%$ at 28,90 and 365 days, respectively. Compared with these results, there were more cases of stroke and higher rates of mortality in the current study $(20 \%$ at 30 days, $28 \%$ at 90 days and $37 \%$ at 365 days). The reasons for the difference may be because we did not exclude patients who had previous stroke and our data are based on hospitalised strokes only. Further, we do not have data on indication of $\mathrm{AP} / \mathrm{AC}$ use prior to stroke or cause of death.

Our results are different in some respects to previously reported studies evaluating the impact of pre-admission $\mathrm{AP} / \mathrm{AC}$ therapy in both ischaemic and haemorrhagic stroke ${ }^{5-8} 2021$ Our study has a much longer follow-up of up to 1 year post event. Furthermore, to date most studies focused on evaluating functional outcomes and stroke severity instead of mortality. Our study also examined ischaemic and haemorrhagic stroke whereas most other studies only focused on one type of stroke. Ideally antithrombotic treatment following the index stroke also needs to be considered. Although we collected the data on AP/ $\mathrm{AC}$ medication at discharge, we were not able to take this into account because discharge AP/AC therapy is dependent on the prior use of these agents (co-linearity issue).

We were not able to explore the mechanism that $\mathrm{AP} / \mathrm{AC}$ therapy might increase the risk of mortality in haemorrhagic stroke and the mechanisms remain unclear. The severity of the vascular event depends on the type of vascular insult and the patient's response to it, which may affect the development and expansion of haematoma and underlying co-pathology. However, previous studies show conflicting results. Some studies suggest that prior AP agents are associated with increased haematoma growth ${ }^{22}$ and mortality, ${ }^{23}$ while another study suggests that they do not affect haemorrhage growth or outcome after intracranial haemorrhage. ${ }^{14}$ This may be because patients' response to brain injury and mortality are likely to be influenced by multiple factors.

The strengths of our study include the large sample size and the fact that the data were collected prospectively. Furthermore, because the UK has a NHS which provides primary (general or 
Table 4 ORs (95\% Cls) of mortality post haemorrhagic stroke based on prior therapy

\begin{tabular}{|c|c|c|c|c|}
\hline Mortality & Unadjusted & Model A & Model B & Model C \\
\hline $0-7$ days, $\mathrm{N}$ & 451 & 451 & 414 & 333 \\
\hline No therapy $\dagger$ & 1.00 & 1.00 & 1.00 & 1.00 \\
\hline Aspirin & 0.99 (0.60 to 1.62 ) & 1.03 (0.62 to 1.71$)$ & 1.24 (0.73 to 2.12 ) & 1.31 (0.64 to 2.68 ) \\
\hline Warfarin & $2.06(1.13 \text { to } 3.79)^{* *}$ & $2.13(1.15 \text { to } 3.96)^{* *}$ & $2.02(1.04 \text { to } 3.89)^{* *}$ & $2.91(1.23 \text { to } 6.89)^{* *}$ \\
\hline $0-30$ days, $\mathrm{N}$ & 451 & 451 & 414 & 378 \\
\hline No therapy $\dagger$ & 1.00 & 1.00 & 1.00 & 1.00 \\
\hline Aspirin & $1.62(1.05 \text { to } 2.50)^{* *}$ & 1.46 (0.93 to 2.29$)$ & $1.70(1.05 \text { to } 2.74)^{* *}$ & $2.36(1.18 \text { to } 4.71)^{* *}$ \\
\hline Warfarin & $1.83(1.02 \text { to } 3.28)^{* *}$ & $1.76(0.97$ to 3.21$)$ & 1.79 (0.95 to 3.38$)$ & $2.37(1.00 \text { to } 5.61)^{* *}$ \\
\hline $0-60$ days, $\mathrm{N}$ & 451 & 451 & 417 & 380 \\
\hline No therapy $\dagger$ & 1.00 & 1.00 & 1.00 & 1.00 \\
\hline Aspirin & $1.62(1.06 \text { to } 2.48)^{* *}$ & $1.40(0.90$ to 2.19$)$ & $1.63(1.02 \text { to } 2.61)^{* *}$ & $2.18(1.10 \text { to } 4.29)^{* *}$ \\
\hline Warfarin & $2.02(1.13 \text { to } 3.61)^{* *}$ & $1.90(1.04 \text { to } 3.46)^{* *}$ & $2.07(1.10 \text { to } 3.89)^{* *}$ & $2.86(1.20 \text { to } 6.84)^{* *}$ \\
\hline 0-90 days, $\mathrm{N}$ & 451 & 451 & 417 & 380 \\
\hline No therapy $\dagger$ & 1.00 & 1.00 & 1.00 & 1.00 \\
\hline Aspirin & $1.80(1.18 \text { to } 2.75)^{*}$ & $1.54(0.99$ to 2.40$)$ & $1.83(1.14 \text { to } 2.93)^{* *}$ & $2.56(1.27 \text { to } 5.13)^{*}$ \\
\hline Warfarin & $1.99(1.11 \text { to } 3.55)^{* *}$ & $1.85(1.02 \text { to } 3.37)^{* *}$ & $2.02(1.08 \text { to } 3.81)^{* *}$ & $2.82(1.16 \text { to } 6.86)^{* *}$ \\
\hline $0-365$ days, $N$ & 451 & 451 & 417 & 380 \\
\hline No therapy $\dagger$ & 1.00 & 1.00 & 1.00 & 1.00 \\
\hline Aspirin & $1.71(1.12 \text { to } 2.61)^{* *}$ & 1.36 (0.86 to 2.13 ) & 1.57 (0.98 to 2.54 ) & 1.67 (0.89 to 3.12 ) \\
\hline Warfarin & $1.87(1.04 \text { to } 3.34)^{* *}$ & 1.67 (0.91 to 3.08$)$ & $1.89(1.00$ to 3.59$)$ & $2.44(1.06 \text { to } 5.62)^{* *}$ \\
\hline
\end{tabular}

family practice), and secondary and tertiary care (hospital medicine) the PAS is a reliable method of mortality ascertainment. We were also able to make adjustments for age, gender, premorbid Rankin score and OCSP classification of stroke, which are important prognostic indicators of mortality. Premorbid Rankin score ${ }^{10}$ and OCSP classifications ${ }^{24}$ have been shown to be major determinants of mortality in stroke.

Our study has limitations. As we used the hospital-based register data some cases of stroke might not have been included, such as patients managed in the community or those who died before admission. Nevertheless, for hospital-based practicing physicians our results may be more generalisable for their patient population. The stroke onset and the time and date of admission may not be exactly the same (usually a couple of hours' difference). However, in a study like ours, with the primary aim of examining the short-term and longer-term outcome with usual clinical reference time points, this will not have an impact on the study findings. Any outlier effect would also be attenuated in a study of such a sample size. The nature of this study is retrospective but identification of patients and data collection occurred prospectively. The stroke severity at onset (eg, National Institutes of Health Stroke Scale (NIHSS) score) is not available. However, we were able to adjust for OCSP, which is indicative of level of stroke severity. Also, we were unable to consider the management patients received after admission. We are not able to quantify the effect of level of AC control (indicated by international normalized ratio (INR)) at the time of stroke onset. It is possible that higher mortality associated with prior use of warfarin in people with haemorrhagic stroke may be solely attributed to cases with higher than the therapeutic range of INR. Another limitation was the small sample size in subsidiary analyses. We also lacked the dose, duration and indication for the use of $\mathrm{AP} / \mathrm{AC}$ drugs. Combinations of $\mathrm{AP}$ and $\mathrm{AC}$ treatment were possible but this is not usually recommended in the UK. There is a possibility of overthe-counter aspirin use, which was not captured in the medical records, resulting in misclassification of some aspirin users as non-users. However, this is unlikely to produce a different direction of the associations observed. We were unable to explore cause-specific mortality, for example cardiovascular versus cancer mortality. It is unclear whether or not the increased mortality may be related to initial stroke severity or a comorbid medical condition associated with antithrombotic use. However, we adjusted premorbid Rankin score as an indicator of disability, which is usually related to comorbidity.

It should be noted that the choice of $\mathrm{AP} / \mathrm{AC}$ in patients who presented with ischaemic stroke depended on the prior $\mathrm{AP} / \mathrm{AC}$ regimen and the aetiology of the stroke (eg, AC for those with atrial fibrillation). We have presented $\mathrm{AP} / \mathrm{AC}$ medication use at the time of hospital discharge for descriptive purposes (see online supplementary tables). Repeating the analyses adjusting for discharge AP/AC status did not alter the results. Further, we were not able to examine the impact of $\mathrm{AP} / \mathrm{AC}$ use or $\mathrm{AC}$ control immediately after stroke, which may have an impact on shortterm and longer-term outcomes and the potential confounding effect cannot be excluded.

Future studies should explore whether the adverse effects of ACs are related to a higher than therapeutic INR level at the time of the stroke. A previous study found that patients taking warfarin on admission with intracranial haemorrhage had double the rate of mortality in a dose-dependent manner. ${ }^{25}$ While some studies support the reversal of coagulopathy on admissions with haemorrhagic stroke, ${ }^{26} 27$ another study suggests that correction of INR does not prevent disease progression. ${ }^{28}$ A study which evaluated various methods to reverse the coagulopathy concluded that randomised trials are required to determine the most effective acute treatment for lasting INR reversal because raised INR was associated with haematoma enlargement. ${ }^{25}$ There is currently a randomised controlled trial underway evaluating the use of platelet transfusion in acute intracerebral haemorrhage. ${ }^{29}$ Whether post-stroke AP/AC choice has an impact on outcome (particularly for ischaemic stroke) requires further evaluation. 


\section{Key messages}

- Prior antiplatelet or anticoagulant use was associated with increased mortality following haemorrhagic stoke but not ischaemic stroke after adjustment for common factors associated with a poor prognosis.

- The reasons for this poor prognosis and potential therapeutic options need exploring in future studies.

In summary, we found that prior $\mathrm{AP} / \mathrm{AC}$ use did not affect mortality after ischaemic stroke. Our study confirmed the worse mortality outcome associated with prior aspirin use and highlighted the potential adverse impact of prior warfarin use on mortality in haemorrhagic stroke. Predictors of poor prognosis in patients with prior AP/AC therapy need further investigation.

Acknowledgements We would like to thank colleagues from the Departments of Medicine for the Elderly and Neurology. We gratefully acknowledge the stroke research team who contributed to data collection and entry.

Contributors PKM conceptualised the article. CSK and JS analysed the data. CSK and PKM prepared the manuscript with critical input from all co-authors. AKM, JFP and PKM are steering group members of the Norfolk and Norwich Stroke Register. PKM is the guarantor.

Funding Norfolk and Norwich Stroke Register is maintained by the Stroke Services, Norfolk and Norwich University Hospital with additional support from the Anglia Stroke and Heart Clinical Network, Eastern Strategic Health Authority.

Disclosures Dr Kwok, Dr Skinner and Dr Metcalfe report no disclosures. Professor Potter serves as Deputy Editor of Age and Ageing. Dr Myint serves as Associate Editor of BMC Health Services Research Journal and is an Editorial Board Member of Open Heart Failure Journal.

\section{Competing interests None.}

Patient consent This is a stroke register which collects information on every adult admitted with an acute stroke which has received institutional approval.

Ethics approval Norfolk and Norwich Hospital Trust Research and Development Committee.

Provenance and peer review Not commissioned; externally peer reviewed.

\section{REFERENCES}

1. Algra A, de Schryver EL, van Gijn J, et al. Oral anticoagulants versus antiplatelet therapy for preventing further vascular events after transient ischaemic attack or minor stroke of presumed arterial origin. Cochrane Database Syst Rev 2001;(4): CD001342

2. Simmons BB, Yeo A, Fung K; American Heart Association; American Stroke Association. Current guidelines on antiplatelet agents for secondary prevention of noncardiogenic stroke: an evidence-based review. Postgrad Med 2010;122:49-53.

3. Diener HC. Primary and secondary prevention of stroke with antiplatelet agents. Curr Pharm Des 2006;12:1293-7.

4. O'Rouke F, Dean N, Akhtar N, et al. Current and future concepts in stroke prevention. CMAJ 2004:170:1123-33.

5. Sanossian N, Saver JL, Rajajee V, et al. Premorbid antiplatelet use and ischaemic stroke outcomes. Neurology 2006;66:3109-23.

6. Wilterdink JL, Bendixen B, Adams HP Jr, et al. Effect of prior aspirin use on stroke severity in the Trial of Org 10172 in Acute Stroke Treatment (TOAST). Stroke 2001;32:2836-40
7. Foerch C, Sitzer M, Steinmetz $\mathrm{H}$, et al. Pretreatment with antiplatelet agents is not independently associated with unfavorable outcome in intracerebral haemorrhage. Stroke 2006;37:2165-7.

8. Thompson BB, Bejot $Y$, Caso $V$, et al. Prior antiplatelet therapy and outcome following intracerebral haemorrhage: a systematic review. Neurology 2010;75:1333-42.

9. Myint PK, Vowler SL, Woodhouse PR, et al. Winter excess in hospital admission, inpatient mortality and length of acute hospital stay in stroke: a hospital database study over six seasonal years in Norfolk, UK. Neuroepidemiology 2007:28:79-85.

10. Farrell B, Godwin J, Richards S, et al. The United Kingdom transient ischaemic attack (UK-TIA) aspirin trial: final results. J Neurol Neurosurg Psychiatry 1991; 54:1044-54.

11. Myint PK, Sankaran P, Musonda P, et al. Performance of CURB-65 and CURB-age in community-acquired pneumonia. Int J Clin Pract 2009:63:1345-50.

12. Saloheimo $\mathbf{P}$, Ahonen $\mathrm{M}$, Juvela $\mathrm{S}$, et al. Regular aspirin-use preceding the onset of primary intracerebral haemorrhage is an independent predictor of death. Stroke 2006:37:129-33

13. Toyoda K, Okada Y, Minematsu K, et al. Antiplatelet therapy contributes to acute deterioration in intracerebral haemorrhage. Neurology 2005;65:1000-4

14. Sansing LH, Messe SR, Cucchiara BL, et al: CHANT Investigators. Prior antiplatelet use does not affect haemorrhage growth or outcome after ICH. Neurology 2009:72:1397-402.

15. de Gans de, Haan RJ, Majoie CB, et al; PATCH Investigators. PATCH: platelet transfusion in cerebral haemorrhage: study protocol for a multicentre, randomised, controlled trial. BMC Neurol 2010;10:19.

16. The ACTIVE Writing Group. Clopidogrel plus aspirin versus oral anticoagulation for atrial fibrillation in the atrial fibrillation clopidogrel trial with irbesartan for prevention of vascular events (ACTIVE W): a randomised controlled trial. Lancet 2006;367:1903-12.

17. Aguilar MI, Hart R, Pearce LA. Oral anticoagulants versus antiplatelet therapy for preventing stroke in patients with non-valvular atrial fibrillation and no history of stroke or transient ischaemic attack. Cochrane Database Syst Rev 2007;(3): CD006186.

18. Heuschmann PU, Kolominsky-Rabas PL, Misselwitz B, et al; German Stroke Registers Study Group. Predictors of in-hospital mortality and attributable risks of death after ischaemic stroke. Arch Intern Med 2004:164:1761-8.

19. Palm F, Urbanek C, Rose S, et al. Stroke incidence and survival in Ludwigshafen am Rhein, Germany: the Ludwigshafen Stroke Study (LuSSt). Stroke 2010;41:1865-70.

20. Greisenegger $\mathbf{S}$, Tentschert $\mathrm{S}$, Weber $\mathrm{M}$, et al. Prior therapy with antiplatelet agents is not associated with outcome in patients with acute ischaemic stroke/TIA J Neurol 2006;253:648-52.

21. Dowlatshahi D, Hakim A, Fang J, et al; Investigators of the Registry of the Canadian Stroke Network. Pre admission antithrombotics are associated with improved outcomes following ischaemic stroke: a cohort from the Registry of the Canadian Stroke Network. Int J Stroke 2009:4:328-34.

22. Broderick JP, Diringer MN, Hill MD, et al. Determinants of intracerebral haemorrhage growth: an exploratory analysis. Stroke 2007;38:1072-5.

23. Creutzfeldt CJ, Weinstein JR, Longstreth WT Jr, et al. Prior antiplatelet therapy platelet infusion therapy and outcome after intracerebral haemorrhage. J Stroke Cerebrovasc Dis 2009:18:221-8.

24. Bamford J, Sandercock P, Dennis M, et al. Classification and natural history of clinical identifiable subtypes of cerebral infarction. Lancet 1991;337:1521-6.

25. Rosand J, Eckman MH, Knudsen KA, et al. The effect of warfarin and intensity of anticoagulation on outcome of intracerebral haemorrhage. Arch Intern Med 2004:164:880-4

26. Goldstein JN, Thomas SH, Frontiero V, et al. Timing of fresh frozen plasma administration and rapid correction of coagulopathy in warfarin-related intracerebra haemorrhage. Stroke 2006;37:151-5.

27. Hutter HB, Schellinger PD, Hartmann M, et al. Hematoma growth and outcome in treated neurocritical care patients with intracerebral haemorrhage related to oral anticoagulant therapy: comparison of acute treatment strategies using vitamin K, fresh frozen plasma, and prothrombin complex concentrates. Stroke 2006; 37:1465-70.

28. Lee SB, Manno EM, Layton KF, et al. Progression of warfarin-associated intracerebral haemorrhage after INR normalization with FFP. Neurology 2006:67:1272-4.

29. Hillborn ME, Juvela SS, Soinne $\mathrm{L}$, et al. Platelet transfusion in acute intracerebral hemorrahge. http://clinicaltrials.gov/ct2/show/NCT00699621 (accessed 31 Jan 2012). 